\title{
BMJ Open PREHAB study: a protocol for a prospective randomised clinical trial of exercise therapy for people living with frailty having cancer surgery
}

Daniel I Mclsaac, ${ }^{1,2}$ Chelsey Saunders, ${ }^{3}$ Emily Hladkowicz, ${ }^{3}$ Gregory L Bryson, ${ }^{1,2}$ Alan J Forster, ${ }^{4,5}$ Sylvain Gagne, ${ }^{1}$ Allen Huang, ${ }^{5,6}$ Manoj Lalu, ${ }^{1,2}$ Luke T Lavallee, ${ }^{2,7}$ Husein Moloo, ${ }^{2,8}$ Julie Nantel, ${ }^{9}$ Barbara Power, ${ }^{5,6}$ Celena Scheede-Bergdahl, ${ }^{10}$ Monica Taljaard, ${ }^{5,11}$ Carl van Walraven, ${ }^{5,12,13}$ Colin J L McCartney ${ }^{1,14}$

To cite: Mclsaac DI

Saunders C, Hladkowicz E, et al. PREHAB study: a protocol for a prospective randomised clinical trial of exercise therapy for people living with frailty having cancer surgery. BMJ Open 2018;8:e022057. doi:10.1136/ bmjopen-2018-022057

- Prepublication history and additional material for this paper are available online. To view these files, please visit the journal online (http://dx.doi. org/10.1136/bmjopen-2018022057).

Received 1 February 2018 Revised 10 April 2018 Accepted 18 May 2018
Check for updates

For numbered affiliations see end of article.

Correspondence to Dr Daniel I Mclsaac; dmcisaac@toh.ca

\section{ABSTRACT}

Introduction Exercise prehabilitation may improve outcomes after surgery. Frailty is a key predictor of adverse postoperative outcomes in older people; the multidimensional nature of frailty makes this a population who may derive substantial benefit from exercise prehabilitation. The objective of this trial is to test the efficacy of exercise prehabilitation to improve postoperative functional outcomes for people living with frailty having cancer surgery with curative intent.

Methods and analysis We will conduct a single-centre, parallel-arm randomised controlled trial of home-based exercise prehabilitation versus standard care among consenting patients $>60$ years having elective cancer surgery (intra-abdominal and intrathoracic) and who are frail (Clinical Frailty Scale $>4$ ). The intervention consists of $\geq 3$ weeks of exercise prehabilitation (strength, aerobic and stretching). The primary outcome is the $6 \mathrm{~min}$ walk test at the first postoperative clinic visit. Secondary outcomes include the short physical performance battery, healthrelated quality of life, disability-free survival, complications and health resource utilisation. The primary outcome will be analysed by intention to treat using analysis of covariance. Outcomes up to 1 year after surgery will be ascertained through linkage to administrative data. Ethics and dissemination Ethical approval has been granted by our ethics review board (Protocol Approval \#2016009-01H). Results will be disseminated through presentation at scientific conferences, through peerreviewed publication, stakeholder organisations and engagement of social and traditional media.

Trial registration number NCT02934230; Pre-results.

\section{INTRODUCTION}

Our population is ageing rapidly, a demographic shift that directly impacts perioperative care. People aged $>60$ years are the fastest growing group of surgical patients, ${ }^{1}$ and experience adverse outcomes at a rate two to four times higher than younger patients. $^{23}$ However, among older surgical patients, research suggests that $25 \%-40 \%$ of

\section{Strengths and limitations of this study}

- Adequately powered and blinded for a patient-centred functional outcome.

- Intervention based on a pragmatic intervention with proven efficacy.

- Complex intervention, possible risk of contamination.

- Feasibility of prehabilitation in older people with frailty before surgery unproven.

adverse outcomes are attributable to the presence of frailty. ${ }^{4-6}$ Frailty is a multidimensional syndrome based on an aggregate susceptibility to adverse health outcomes due to age-related and disease-related deficits that accumulate across multiple domains. ${ }^{78}$ Independent of age, gender and other confounders, people with frailty have significantly higher rates of postoperative morbidity, mortality and healthcare resource use. People living with frailty are also significantly more likely to develop a new disability after elective surgery than older people without frailty. ${ }^{9}$

Despite the growing observational literature that links the presence of preoperative frailty with adverse outcomes across different surgical procedures, the literature evaluating interventions to improve the postoperative outcomes of people living with frailty is sparse. ${ }^{6}$ Frailty-related risk is manifest through vulnerability to stressors. ${ }^{7}$ Surgery induces substantial physiological stress, and some data suggest that people with frailty experience a significantly increased risk of early mortality (over 30 times higher than non-frail patients on postoperative day 3$).{ }^{10}$ These findings support the hypothesis that the limited physical reserve of frail patients may contribute to their risk of adverse outcomes. Therefore, interventions that target the 
physical reserve of people with frailty may contribute to improved outcomes. Exercise training in non-surgical people with frailty improves functional capacity, muscle strength and may decrease frailty itself. ${ }^{11-13}$ In particular, structured multicomponent programmes demonstrate superior outcomes to other types of programmes in older people with frailty. ${ }^{12}$ Exercise prehabilitation (preoperative exercise training) of people without frailty having colorectal surgery improves postoperative function when compared with postoperative exercise alone,${ }^{14}$ an effect that may be especially pronounced in people who are older or who have multimorbidity. A recent randomised trial of personalised prehabilitation in high-risk older people having abdominal surgery reduced complications by $50 \% .{ }^{15}$ Therefore, we hypothesise that exercise prehabilitation may be an appropriate intervention to improve the postoperative outcomes of people living with frailty.

The primary objective of this study is to test the efficacy of home-based exercise prehabilitation for older people with frailty having elective surgery with curative intent for intra-abdominal or intrathoracic cancer, to improve postoperative function as measured by the $6 \mathrm{~min}$ walk test (6-MWT) at their first postoperative clinic visit in a parallel-arm superiority trial with equal allocation between arms. Our secondary objective is to measure this intervention's efficacy in improving other important outcomes, including patient-reported and health system measures.

\section{METHODS AND ANALYSIS}

\section{Study design and setting}

We will conduct a single-centre, parallel-arm randomised controlled trial of home-based exercise prehabilitation versus standard perioperative care in people living with frailty undergoing elective surgery for intra-abdominal and intrathoracic known or suspected cancer at The Ottawa Hospital (TOH). This will be a superiority trial to test the hypothesis that home-based exercise prehabilitation will result in improved postoperative functional outcomes compared with standard care plus a generic activity guide. This protocol is reported in keeping with the Standard Protocol Items: Recommendations for Intervention Trials guidelines. ${ }^{16} \mathrm{TOH}$ is a 900 -bed tertiary care academic health sciences centre serving a catchment area of 1.2 million people. TOH is the regional cancer referral centre for the Eastern portion of the Canadian province of Ontario. On average, intra-abdominal and patients with intrathoracic cancer are seen 4 weeks prior to surgery (as this is a provincial benchmark for cancer care). Research ethics board (REB) approval has been granted by the study centre.

\section{Eligibility criteria}

All consenting patients 60 years or older who are: scheduled to undergo elective surgery for intra-abdominal and intrathoracic cancer (colorectal, thoracic, hepatobiliary or urological); able to communicate in French or English; willing to participate in home-based exercise and identified with frailty based on the Clinical Frailty Scale (CFS; score of $\geq 4 / 9$ ) will be included. The CFS is a nine-point global frailty scale based on clinical evaluation and judgement of an individual's mobility, energy, physical activity and function. ${ }^{8}$ The CFS is highly correlated $(\rho-0.80)$ with the Canadian Study of Health and Ageing Frailty Index. A multitude of frailty instruments exist to diagnose frailty. We have chosen the CFS as it is easily administered, has excellent inter-rater reliability and has been shown to accurately identify older patients at high risk of adverse outcomes in a variety of acute-care settings. ${ }^{8} 1718$ The CFS will be administered by trained clinicians and clinical researchers.

\section{Intervention}

The intervention will be a home-based total-body exercise training programme (exercise prehabilitation), based on a protocol with proven efficacy in improving the function of people without frailty in less than 4 weeks before surgery $^{1419}$ (see online supplementary methods 1 ). All intervention group participants will be exposed to at least 3 weeks of exercise, as previous use of this exercise protocol was shown to be efficacious with a median of 24 days participation, ${ }^{14}$ and because provincial benchmarks require less than 4 weeks from diagnosis to cancer surgery. However, because some individuals will undergo neoadjuvant chemotherapy, diagnosis to surgery time frames may vary. Therefore, we will take a pragmatic approach and allow for variable exposure periods for the intervention. Exercise prehabilitation will consist of three components: (1) strength training, (2) aerobic exercise and (3) flexibility. Exercise prehabilitation will be prescribed as 1-hour sessions performed a minimum of 3 times per week. Intervention group patients will also be provided with standard nutritional advice. In addition to paper-based materials outlining the exercise prehabilitation programme, participants will have an individualised teaching session at the time of recruitment as well as being provided with a take home video. Furthermore, activity logs and weekly phone calls will be used to encourage and measure compliance and to answer questions.

The strength training component consists of 1 set of 10 repetitions of 10 exercises. The exercises include: push ups, seated rows, chest fly, deltoid lift, bicep curls, triceps extensions, chair squats, hamstring curls, standing calf raises and abdominal crunches. The participants will be provided with an elastic band in order to complete these exercises at home. In addition, participants will be encouraged to modify the exercises based on ability. The aerobics component consists of the participants' choice of cardio (eg, walking, biking or swimming) for $20 \mathrm{~min}$ at moderate intensity. Lastly, the flexibility component consists of six stretches, each to be held for $20 \mathrm{~s}$, done for two repetitions. The stretches target the chest, arms, legs and truck.

Participants randomised to the control group will receive WHO Global Recommendations for Physical Activity for Health for people 60 years and above pamphlet, as well as 
Canada's Food Guide. Both groups will receive pedometers to track their daily step count before surgery.

All other preoperative, intraoperative and postoperative care will be at the discretion of each patient's care team for both intervention and control arm participants. Specifically, intraoperative anaesthesia interventions and intraoperative and postoperative surgical care will be at the discretion of treating physicians.

\section{Outcomes}

The primary outcome will be postoperative functional capacity, measured using the 6-MWT. The 6-MWT will be administered at baseline and at the first postoperative clinic visit. The 6-MWT has been widely used for preoperative and postoperative evaluation and for measuring the response to therapeutic interventions for pulmonary and cardiac disease.$^{20} \mathrm{~A}$ clinically relevant difference in this outcome is a change of $25 \mathrm{~m}$ walked over 6 min. ${ }^{19} 21$ All patients will perform a standardised, self-paced 6-MWT test in a $30 \mathrm{~m}$ long corridor. They will be instructed to walk as far as possible for $6 \mathrm{~min}$. Patients will be allowed to stop at any time but will be encouraged to restart as soon as possible. Covered distance after 6 min will be measured to the nearest metre.

Secondary outcomes will reflect four specific domains: (1) function, (2) patient-reported health outcomes and complications, (3) healthcare resource utilisation and (4) patient experience with exercise prehabilitation. Outcome assessment windows are shown in figure 1 .

Secondary functional outcomes will be assessed using the short physical performance battery (SPPB), measured at baseline and at the first postoperative clinic visit. This is a validated, objective assessment which evaluates lower extremity functioning in older individuals through assessment of balance, gait speed and lower limb functional strength. ${ }^{22-24}$ Individuals unable to complete a task, receive a score of 0 .

Patient-reported health-related quality of life (HRQoL) using the EuroQoL five-dimensional questionnaire $(\text { EQ-5D })^{25}$ (five-level version) will be measured at baseline, first clinic visit and 90 days after surgery. The EQ-5D assesses domains of self-perceived mobility, self-care, usual activity participation, pain/discomfort and anxiety depression, as well as a $0-100$ point scale relating the person's current health status to their best imaginable status. Patient-reported disability will be measured using WHO Disability Assessment Schedule V.2.0 instrument, ${ }^{26}$ a 12-item, 30-day look-back multidimensional disability scale that is validated in a variety of disease states, including surgery. ${ }^{26-30}$ Disability scores will be measured at baseline, first clinic follow-up and 90 days after surgery. Disability-free survival will be assessed at 90 days, based on an individual surviving to 90 days after surgery without developing a new disability. ${ }^{31}$ Complications will be identified during the index hospitalisation using the Postoperative Morbidity Survey. ${ }^{32}$

Healthcare resource utilisation measures will include length of hospital stay, discharge to an institution, readmissions within 30 days of discharge and total healthcare costs (using a validated algorithm in our administrative data $\left.^{33}\right)$.

Patient experience with exercise prehabilitation will be examined. The validated 10 -item version of the positive and negative affect schedule will be used to measure participants' feelings and emotions after exercise. ${ }^{34} \mathrm{~A}$ subset of questions taken from the Basic Psychological Needs in Exercise scale will be used to measure participants' competence and autonomy felt in relation to their participation in the exercise prehabilitation programme. ${ }^{35}$ We will conduct semistructured interviews using an interview guide informed by the Theoretical Domains Framework $^{36}$ to provide insight into the barriers and facilitators to performing exercise prehabilitation in this population. All participants will be asked how likely they would be to recommend the prehabilitation programme to a similar patient going for a similar surgery as themselves.

\section{Sample size}

To detect a clinically important $25 \mathrm{~m}$ difference in the mean 6-MWT between study arms, using a two-sided two-sample equal-variance t-test at the $5 \%$ level of significance with $80 \%$ power, and assuming an SD of $55 \mathrm{~m}$ based on a previously published trial, ${ }^{14}$ we will require 77 patients per arm. This sample size calculation is conservative as it does not account for the increased efficiency due to adjustment for the baseline 6-MWT in an analysis of covariance (ANCOVA). Assuming a conservative estimate of a 0.5 correlation with baseline 6-MWT, power to detect the minimum important difference increases to $90 \%$. To account for up to $20 \%$ attrition, we will enrol 100 patients per arm.

\section{Recruitment}

Patients will be recruited from our hospital's Cancer Assessment Center. Following cancer diagnosis, patients are seen by a surgeon $3-4$ weeks prior to their scheduled operation. Following surgical assessment and confirmation of the decision to operate, patients who consent to consideration for research contact, and who meet all inclusion criteria except for frailty score, will be assessed by a trained clinician or clinical assistant using the CFS. ${ }^{8}$ Patients who score $\geq 4 / 9$ on the CFS will then be offered the opportunity to provide written informed consent.

\section{Patient and public involvement}

A James Lind Alliance Research Priority Partnership has identified the role of exercise and care of older people having surgery as 2 of the top 10 priorities in perioperative research. ${ }^{37}$ These priorities directly informed our research question. We also ensured that our study was powered to address a patient-centred primary outcome which also reflected function, an outcome of key importance for older people. ${ }^{38}$ Patients were not, however, directly involved in design, recruitment or conduct of the study. As described in the outcomes section above, patient experience with the intervention will be measured 


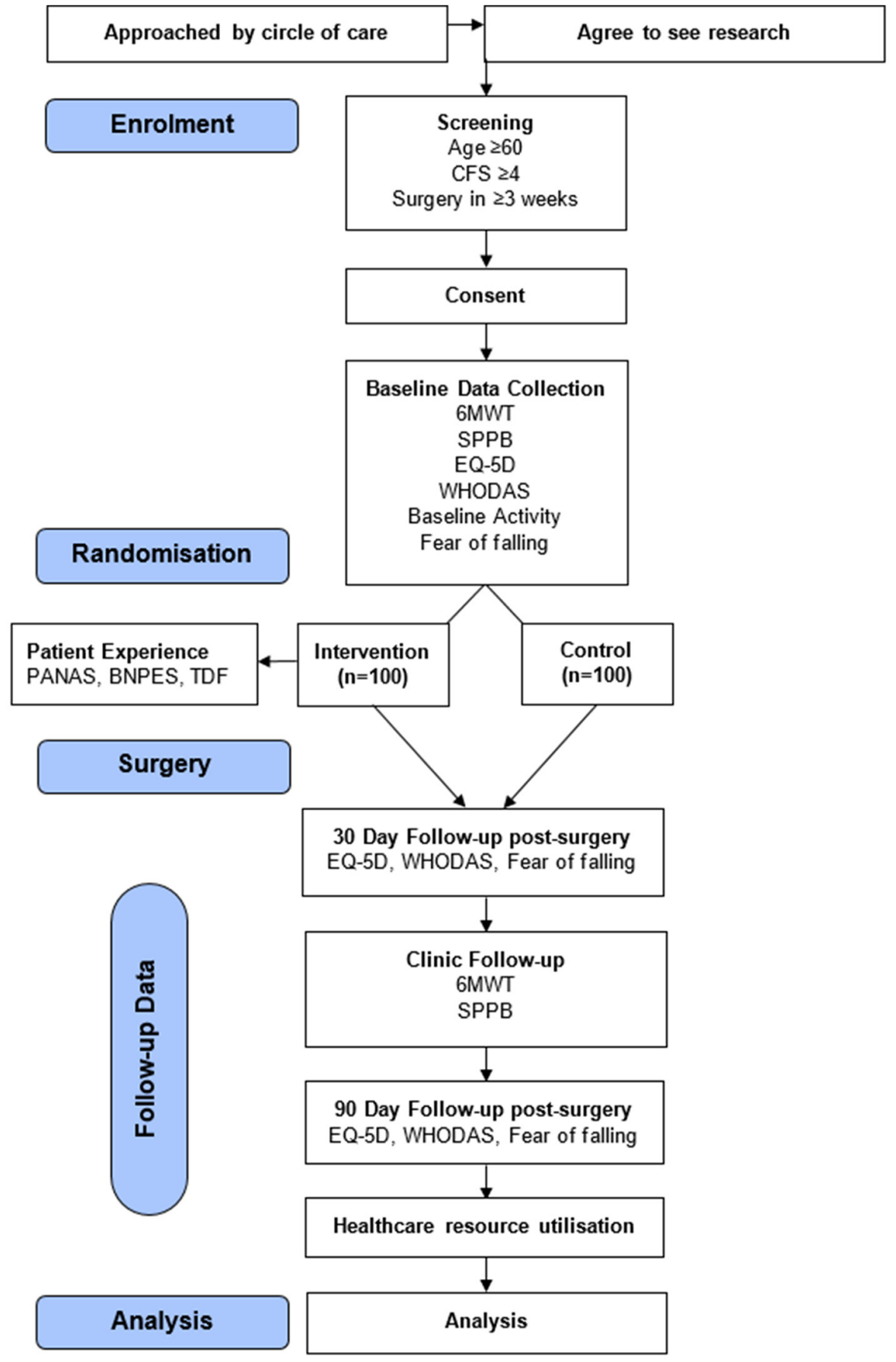

Figure 1 Study flow. BPNES, Basic Psychological Needs in Exercise Scale; CFS, Clinical Frailty Scale; 6-MWT, 6 min walk test; PANAS, Positive and Negative Affect Schedule; SPPB, short physical performance battery; TDF, Theoretical Domains Framework; WHODAS, WHO Disability Assessment Schedule measuring Disability-Free Survival; EQ-5D: Health-Related Quality of Life Measure; Baseline Activity Questionnaire; Fear of Falling Questionnaire; Healthcare resource utilisation: length of hospital stay, discharge to an institution, readmissions within 30 days of discharge and total healthcare costs.

quantitatively and qualitatively. Study results will be disseminated to participants through social media and our hospital patient and family advisory council.

\section{Assignment of the intervention}

The random allocation sequence will be computer generated by the study biostatistician using permuted blocks of randomly varying lengths, stratified on planned open versus minimally invasive surgery. Study personnel will access the randomisation sequence via a central secure internet-based application to ensure adequate allocation concealment.

\section{Blinding}

Clinicians and outcome assessors will be blinded to treatment allocation. All participants will be informed that they are being enrolled in a study to increase their level of physical activity prior to surgery. Control arm patients 
will be provided WHO activity pamphlet and encouraged to be active prior to surgery; however, treatment status cannot be fully concealed to participants.

\section{Data collection and management}

Data will be collected in three ways. All preoperative functional assessments, and demographics will be collected by trained study personnel using a secure, iPad-based application that has been specifically designed for this study. Postoperative functional outcomes, HRQoL and disability will also be collected with this application, either in person or by phone. Patients will keep a daily activity log during the exercise prehabilitation phase. Length of stay, discharge disposition, readmissions and healthcare costs and mortality will be calculated through linkage to our hospital data warehouse and provincial health administrative data system, which contain validated measured of these outcomes. All study data will be stored on a secure server in our hospital data warehouse in a privacy legislation compliant manner. Patient diaries will be entered into the data collection system and stored in the same way; paper copies will be maintained in an appropriately locked and secured filing cabinet. The principal investigator will have access to the blinded data set. Data linked to our provincial health administrative data system will be stored and managed according to specific privacy legislation which governs use of this data.

\section{Data analysis}

All outcomes will be analysed according to intention to treat principles. Descriptive statistics (mean and SD for continuous variables or median and IQR for skewed distributions, and frequency and proportion for categorical variables) will be used to compare characteristics of participants at baseline.

The primary outcome will be measured at baseline and first postdischarge clinic visit. The response at the first postdischarge visit will be analysed using linear regression analysis with the baseline measure entered as a covariate (ie, using ANCOVA). The model will include the stratification factors (planned open vs minimally invasive surgery), and prespecified covariates: age, sex, surgery type, preoperative chemo, American Society of Anesthesiology score and frailty score. The intervention effect will be estimated using the adjusted least square mean difference between arms and presented together with 95\% CI. Every effort will be made to avoid missing outcome data; nevertheless, to assess the potential for differential attrition, the characteristics of patients dropping out will be compared between arms, as well as to the characteristics of patients completing the study. To adjust for potential bias due to attrition under a missing at random mechanism, the regression model will include baseline characteristics found to be associated with attrition. Sensitivity analyses will additionally be carried out to examine the potential impact of non-random missingness under a pattern-mixture model ${ }^{39}$ We will also perform a per-protocol analysis with $\geq 80 \%$ compliance based on activity logs (ie, completion of $\geq 80 \%$ of prescribed exercise sessions) considered as adherent to the protocol.

The SPPB will be analysed in the same manner as the primary outcome. Secondary outcomes measured at $>2$ time points (EQ-5D, disability) will be analysed using repeated measures linear regression. Binary secondary outcomes (disability-free survival, complications, readmissions and institutional discharge) will be analysed using logistic regression. Time to hospital discharge will be analysed using Cox regression with in-hospital mortality as a competing risk. Total healthcare costs will be compared using a generalised linear model with gamma distributed errors and a $\log$ link to account for the skewed nature of cost data. Overall survival will be analysed using Cox regression. All secondary analyses will include the same covariates as described for the primary outcome.

Patient experience with exercise prehabilitation data will be analysed descriptively (mean and SD for continuous variables or median and IQR for skewed distributions, and frequency and proportion for categorical variables). Interview data will be transcribed and then coded in duplicate to identify responses relevant to the theoretical domains. The coded data will then be used to identify consistent belief statements, which represent an underlying theme that impacts behaviour. The frequency of each belief statement will be calculated.

\section{ETHICS AND DISSEMINATION}

Each participant will be given the opportunity to read, consider and ask questions about the information in the informed consent form. The trained research assistant must obtain written informed consent (see online supplementary material) from the participant before any study procedures occur. Any modifications to the protocol which may impact on the conduct of the study, potential benefit of the patient or may affect patient safety, including changes of study objectives, study design, patient population, sample sizes, study procedures or significant administrative aspects will require a formal amendment to the protocol. Such amendments will be reviewed and approved by the local REB. All items from WHO Trial Registration Data Set can be viewed as an online supplementary file.

\section{Confidentiality}

Patients' data will be anonymised using a study identification number that will be stored using a protected file separated from the research data. This file will be stored on a secured hospital server where only the researchers in this study will have access to the research data.

\section{Monitoring}

A Data and Safety Monitoring Board (DSMB) has been established. During the period of recruitment, interim analyses and safety outcomes will be supplied in confidence to the DSMB, along with any other analyses that the committee may request. The purpose of the DSMB is 
to protect participant safety, safeguard the credibility and integrity of the trial for subjects, and to ensure the timely conclusion of the trial so its results can be disseminated.

All adverse events that occur after enrolment during in-person data collection and throughout the exercise prehabilitation period will be documented. Serious adverse events that the principal investigator deems related to the study protocol will be reported to the REB as soon as possible. Local protocols mandate that reporting occur within 7 days if the study-related serious adverse event is unexpected and involves greater risk. Adverse events related to the participants underlying cancer and related treatment will not be collected as part of this study.

\section{Dissemination}

Results will be disseminated through presentation at scientific conferences, through peer-reviewed publication, stakeholder organisations and engagement of social and traditional media.

\section{DISCUSSION}

Older people living with frailty represent a growing and high-risk stratum of the perioperative population. Interventions to improve the outcomes of older surgical patients living with frailty are urgently needed. ${ }^{6}$ This prospective randomised clinical trial will address multiple knowledge gaps in the perioperative frailty literature. The findings will provide novel insights into improving patient-centred and system-centred outcomes. Due to the increasing prevalence of older adults living with frailty in the perioperative setting, and associated adverse outcomes, interventions tailored for, and tested in, this population are a priority.

Several studies have demonstrated that exercise prehabilitation in patients going for abdominal or cancer surgery may reduce adverse events and improve postoperative function ${ }^{14}{ }^{15}$ However, to our knowledge, no studies have measured the impact of exercise prehabilitation on postoperative outcomes in surgical patients living with frailty. Furthermore, studies that have evaluated the impact of exercise prehabilitation on outcomes in any surgical patients have typically been underpowered for many important outcomes (median sample size 54 participants), and at significant risk of several biases. ${ }^{16}$ Additionally, most studies have focused on younger and relatively well patients (mean age 63 years), populations who may be less likely to derive benefit from exercise prehabilitation. ${ }^{40-42}$ In several studies of surgical patients with an average age $<65$ years, function and HRQoL were not consistently improved. ${ }^{41-43}$ In contrast, a recent study of high-risk older people (mean age 71 years) demonstrated improvements in postoperative function and a decreased rate of complications. ${ }^{15}$ In keeping with these findings, our study focuses on individuals with lower baseline functional capacity (ie, frailty). Such individuals, we hypothesise, may have the most to gain from preoperative exercise. Our study is also adequately powered to detect an important difference in a patient-centred outcome and has been designed to be at low risk of bias through use of robust methods of allocation concealment, blinding and outcome adjudication. Furthermore, collection of our more distal patient-reported secondary outcome measures (disability, HRQoL) will allow for accurate sample size estimations for future, multicentre studies of exercise prehabilitation in older people living with frailty.

\section{Limitations}

Exercise therapy is a complex intervention that we will test in a population of patients who tend to have low baseline activity levels; feasibility of the intervention in this population has not been evaluated previously. This could lead to issues with protocol compliance that could lead to underestimation of the efficacy of the intervention, although our study processes have been designed to minimise this risk through regular compliance and support calls to intervention arm participants and per-protocol analyses. There is also the possibility that new introduction of increased activity in previously sedentary and functionally limited individuals could cause adverse effects such as falls or myocardial ischaemia. However, in addition to monitoring for these potential adverse effects, exercise will be introduced in a graded fashion and supported by regular calls to advance activity as appropriate. Furthermore, participants will have already been deemed fit for major surgery, which typically involves tolerance of four metabolic equivalents (consistent with moderate intensity activity).$^{44}$ Contamination could also occur between the control and intervention groups as physicians and nurses practising at the site may be influenced by the research study and may incorporate exercise prescription as part of their practice. To mitigate the risk that knowledge of which arm a patient has been allocated to influence their behaviour, participants in both study arms will be told that they are being enrolled in an exercise trial and age-appropriate activity guidelines are being provided to control arm participants. While this could reduce the relative impact of the intervention through increasing activity levels in the control group, we believe that the reduction in the risks of bias from knowledge of their allocation status more than outweighs the concern about attenuation of the intervention effect. Furthermore, relative activity levels between study arms will be monitored though use of a pedometer in all study participants.

\section{CONCLUSION}

In summary, we propose to evaluate the efficacy of a home-based exercise prehabilitation programme in frail elderly patients in preparation for cancer surgery to improve postoperative function. We plan to disseminate the results of this randomised clinical trial in peer-reviewed journals and presentations at scientific 
meetings. The results of this study will inform current perioperative practice and will provide direction for future research.

\section{Author affiliations}

${ }^{1}$ Department of Anesthesiology and Pain Medicine, The Ottawa Hospital, University of Ottawa, Ottawa, Ontario, Canada

${ }^{2}$ Clinical Epidemiology Program, The Ottawa Hospital Research Institute, Ottawa, Ontario, Canada

${ }^{3}$ Department of Anesthesiology and Pain Medicine, The Ottawa Hospital Research Institute, Ottawa, Ontario, Canada

${ }^{4}$ Division of General Internal Medicine, The Ottawa Hospital, University of Ottawa, Ottawa, Ontario, Canada

${ }^{5}$ Clinical Epidemiology Program, The Ottawa Hospital Research Institute, Ottawa, Ontario, Canada

${ }^{6}$ Division of Geriatric Medicine, The Ottawa Hospital, University of Ottawa, Ottawa, Ontario, Canada

${ }^{7}$ Division of Urology, The Ottawa Hospital, University of Ottawa, Ottawa, Ontario,

Canada

${ }^{8}$ Division of General Surgery, The Ottawa Hospital, University of Ottawa, Ottawa, Ontario, Canada

${ }^{9}$ School of Human Kinetics, Faculty of Health Sciences, University of Ottawa, Ottawa, Canada

${ }^{10}$ Department of Kinesiology and Physical Education, McGill University, Montreal, Quebec, Canada

${ }^{11}$ School of Epidemiology, Population Health and Preventative Medicine, University of Ottawa, Ottawa, Ontario, Canada

${ }^{12}$ Department of Medicine, The Ottawa Hospital, University of Ottawa, Ottawa, Ontario, Canada

${ }^{13}$ Institute for Clinical Evaluative Sciences, Ottawa, Ontario, Canada

${ }^{14}$ The Ottawa Hospital Research Institute, Ottawa, Ontario, Canada

Acknowledgements We would like to acknowledge Kristin Dorrance for her insight into the patient experience component of the protocol.

Contributors DIM is the principal investigator. DIM was involved in the conception and design of the study and initial draft of the protocol. CS contributed to the draft of the protocol. CS and EH participated in the implementation of the study (submissions to ethics committee, daily management of the trial) and EH critically reviewed the protocol and its contents. JN provided her expertise in kinesiology and contributed to the knowledge needed for the prehabilitation program. HM, LTL, ML, GLB, AH and SG provided their experience in epidemiology, quality improvement and clinical practice to the study design and procedures, and writing of the protocol. BP provided her knowledge for the care of ageing patients and provided insight into the intervention design. AJF contributed heavily to the study design and methodology. CS-B contributed to the study design and the details of the intervention and control groups. CrW was involved in the study methodology and provided his expertise on data linkage. MT developed the analysis plan. CJLM provided mentorship oversight and helped draft the protocol. All authors read and approved the final protocol.

Funding This work was supported by the International Anesthesia Research Society and the University of Ottawa Anesthesiology Research Grant.

Competing interests LTL reports grants from Sanofi, personal fees from Sanofi and Ferring, outside the submitted work. CJLM reports other from Teleflex Medical, outside the submitted work.

Patient consent Not required.

Ethics approval Prior to the commencement of the study, the protocol was presented to the independent ethics committee of The Ottawa Health Sciences Network-Research Ethics Board. Ethics approval was subsequently granted (Protocol \#20160091-01H).

Provenance and peer review Not commissioned; externally peer reviewed.

Open access This is an open access article distributed in accordance with the Creative Commons Attribution Non Commercial (CC BY-NC 4.0) license, which permits others to distribute, remix, adapt, build upon this work non-commercially, and license their derivative works on different terms, provided the original work is properly cited and the use is non-commercial. See: http://creativecommons.org/ licenses/by-nc/4.0/ (c) Article author(s) (or their employer(s) unless otherwise stated in the text of the article) 2018. All rights reserved. No commercial use is permitted unless otherwise expressly granted.

\section{REFERENCES}

1. Etzioni DA, Liu JH, O'Connell JB, et al. Elderly patients in surgical workloads: a population-based analysis. Am Surg 2003;69:961-5.

2. Hamel MB, Henderson WG, Khuri SF, et al. Surgical outcomes for patients aged 80 and older: morbidity and mortality from major noncardiac surgery. J Am Geriatr Soc 2005;53:424-9.

3. Turrentine FE, Wang H, Simpson VB, et al. Surgical risk factors, morbidity, and mortality in elderly patients. J Am Coll Surg 2006;203:865-77.

4. Beggs T, Sepehri A, Szwajcer A, et al. Frailty and perioperative outcomes: a narrative review. Canadian Journal of Anesthesial Journal canadien d'anesthésie 2015;62:143-57.

5. Kim DH, Kim CA, Placide S, et al. Preoperative Frailty Assessment and Outcomes at 6 Months or Later in Older Adults Undergoing Cardiac Surgical Procedures: A Systematic Review. Ann Intern Med 2016;165:650-60.

6. Mclsaac DI, Jen T, Mookerji N, et al. Interventions to improve the outcomes of frail people having surgery: A systematic review. PLoS One 2017;12:e0190071.

7. Fried LP, Ferrucci L, Darer J, et al. Untangling the Concepts of Disability, Frailty, and Comorbidity: Implications for Improved Targeting and Care. J Gerontol A Biol Sci Med Sci 2004;59:M255-M263.

8. Rockwood K, Song X, MacKnight C, et al. A global clinical measure of fitness and frailty in elderly people. CMAJ 2005;173:489-95.

9. Mclsaac DI, Bryson G, Forster AJ, et al. Comparative assessment of two frailty instruments to predict patient-reported disability after elective noncardiac surgery. BMC Anesthesiol 2017;16:111.

10. Mclsaac DI, Bryson GL, van Walraven C. Association of Frailty and 1-Year Postoperative Mortality Following Major Elective Noncardiac Surgery: A Population-Based Cohort Study. JAMA Surg 2016;151:538.

11. de Labra C, Guimaraes-Pinheiro C, Maseda A, et al. Effects of physical exercise interventions in frail older adults: a systematic review of randomized controlled trials. BMC Geriatr 2015;15:154.

12. Theou O, Stathokostas L, Roland KP, et al. The effectiveness of exercise interventions for the management of frailty: a systematic review. J Aging Res 2011;2011:1-19.

13. Tarazona-Santabalbina FJ, Gómez-Cabrera MC, Pérez-Ros $P$, et al. A Multicomponent Exercise Intervention that Reverses Frailty and Improves Cognition, Emotion, and Social Networking in the Community-Dwelling Frail Elderly: A Randomized Clinical Trial. J Am Med Dir Assoc 2016;17:426-33.

14. Gillis C, Li C, Lee L, et al. Prehabilitation versus rehabilitation: a randomized control trial in patients undergoing colorectal resection for cancer. Anesthesiology 2014;121:937-47.

15. Barberan-Garcia A, Ubré M, Roca J, et al. Personalised Prehabilitation in High-risk Patients Undergoing Elective Major Abdominal Surgery: A Randomized Blinded Controlled Trial. Ann Surg 2018;267:50-6.

16. Chan AW, Tetzlaff JM, Altman DG, et al. SPIRIT 2013 statement: defining standard protocol items for clinical trials. Ann Intern Med 2013;158:200.

17. Wallis SJ, Wall J, Biram RW, et al. Association of the clinical frailty scale with hospital outcomes. QJM 2015;108:943-9.

18. Bagshaw SM, Stelfox HT, McDermid RC, et al. Association between frailty and short- and long-term outcomes among critically ill patients: a multicentre prospective cohort study. Can Med Assoc J 2014;186:E95-E102.

19. Li C, Carli F, Lee L, et al. Impact of a trimodal prehabilitation program on functional recovery after colorectal cancer surgery: a pilot study. Surg Endosc 2013;27:1072-82.

20. ATS statement: guidelines for the six-minute walk test. Am J Respir Crit Care Med 2002;166:111-7.

21. Carli F, Charlebois $P$, Stein B, et al. Randomized clinical trial of prehabilitation in colorectal surgery. Br J Surg 2010;97:1187-97.

22. Guralnik JM, Simonsick EM, Ferrucci L, et al. A short physical performance battery assessing lower extremity function: association with self-reported disability and prediction of mortality and nursing home admission. J Gerontol 1994;49:M85-M94.

23. Freire AN, Guerra RO, Alvarado B, et al. Validity and reliability of the short physical performance battery in two diverse older adult populations in Quebec and Brazil. J Aging Health 2012;24:863-78. 
24. Volpato S, Cavalieri M, Sioulis F, et al. Predictive value of the Short Physical Performance Battery following hospitalization in older patients. J Gerontol A Biol Sci Med Sci 2011;66:89-96.

25. Bansback N, Tsuchiya A, Brazier J, et al. Canadian valuation of EQ$5 \mathrm{D}$ health states: preliminary value set and considerations for future valuation studies. PLoS One 2012;7:e31115.

26. Shulman MA, Wallace S, Ponsford J, et al. Measurement of Disability-free Survival after Surgery. 2015;3:524-36.

27. Schlote A, Richter M, Wunderlich MT, et al. WHODAS II with people after stroke and their relatives. Disabil Rehabil 2009;31:855-64.

28. Wolf AC, Tate RL, Lannin NA, et al. The World Health Organization Disability Assessment Scale, WHODAS II: reliability and validity in the measurement of activity and participation in a spinal cord injury population. J Rehabil Med 2012;44:747-55.

29. Soberg HL, Finset A, Roise O, et al. The trajectory of physical and mental health from injury to 5 years after multiple trauma: a prospective, longitudinal cohort study. Arch Phys Med Rehabil 2012;93:765-74.

30. Garin O, Ayuso-Mateos JL, Almansa J, et al. Validation of the "World Health Organization Disability Assessment Schedule, WHODAS-2" in patients with chronic diseases. Health Qual Life Outcomes 2010;8:51.

31. Shulman MA, Myles PS, Chan MT, et al. Measurement of disabilityfree survival after surgery. Anesthesiology 2015;122:524-36.

32. Grocott MP, Browne JP, Van der Meulen J, et al. The Postoperative Morbidity Survey was validated and used to describe morbidity after major surgery. J Clin Epidemiol 2007;60:919-28.

33. Wodchis W, Bushmeneva K, Nikitovic M, et al. Guidelines on PersonLevel Costing Using Administrative Databases in Ontario. Toronto: ON, 2013.

34. Mackinnon A, Jorm AF, Christensen $\mathrm{H}$, et al. A short form of the Positive and Negative Affect Schedule: evaluation of factorial validity and invariance across demographic variables in a community sample. Pers Individ Dif 1999;27:405-16.
35. Vlachopoulos SP, Michailidou S. Development and Initial Validation of a Measure of Autonomy, Competence, and Relatedness in Exercise: The Basic Psychological Needs in Exercise Scale. Meas Phys Educ Exerc Sci 2006;10:179-201.

36. Atkins L, Francis J, Islam R, et al. A guide to using the Theoretical Domains Framework of behaviour change to investigate implementation problems. Implement Sci 2017:12:1-18.

37. The Anaesthesia and Perioperative Care Priority Setting Partnership. 2015 http://niaa.org.uk/Results\#pt (27 Aug 2015).

38. Fried TR, Bradley EH, Towle VR, et al. Understanding the treatment preferences of seriously ill patients. N Engl J Med 2002;346:1061-6.

39. Little RJ, D'Agostino R, Cohen ML, et al. The prevention and treatment of missing data in clinical trials. N Engl $J$ Med 2012;367:1355-60

40. Marmelo F, Rocha V, Gonçalves D. The impact of prehabilitation on post-surgical complications in patients undergoing non-urgent cardiovascular surgical intervention: Systematic review and metaanalysis. Eur J Prev Cardiol 2018;25:404-17.

41. Ferrara PE, Rabini A, Maggi L, et al. Effect of pre-operative physiotherapy in patients with end-stage osteoarthritis undergoing hip arthroplasty. Clin Rehabil 2008;22:977-86.

42. D'Lima DD, Colwell CW, Morris BA, et al. The effect of preoperative exercise on total knee replacement outcomes. Clin Orthop Relat Res 1996;326:174-82.

43. Bäck $\mathrm{M}$, Wennerblom $\mathrm{B}$, Wittboldt $\mathrm{S}$, et al. Effects of high frequency exercise in patients before and after elective percutaneous coronary intervention. Eur J Cardiovasc Nurs 2008;7:307-13.

44. Fleisher LA, Beckman JA, Brown KA, et al. ACC/AHA 2007 guidelines on perioperative cardiovascular evaluation and care for noncardiac surgery: executive summary: a report of the American College of Cardiology/American Heart Association Task Force on Practice Guidelines (Writing Committee to Revise the 2002 Guidelines on Perioperative Cardiovascular Evaluation for Noncardiac Surgery). Anesth Analg 2008;106:685-712. 